\title{
Passive range estimation using subarray parallax
}

\author{
Melvin J. Hinich \\ Virginia Polytechnic Institute and State .University, Blacksburg, Virginia 24061 \\ (Received 14 October 1978; revised 8 January 1979) \\ Consider a large aperture linear array which is detecting coherent radiation from a source. Suppose that \\ the array is processed as a string of subarrays consisting of adjacent sensors. Assume that the signal is a \\ plane wave whose coherence distance is of the order of the subarray lengths. There is a systematic \\ difference among the subarray bearing estimates due to parallax. A statistical method for using the \\ parallax effect to estimate range is presented. Approximate expressions for the variance and bias of the \\ estimator are derived under the assumption that the gain of each subarray is large. These theoretical \\ results are compared with values obtained using artificial data for several parameter values.
}

PACS numbers: $43.60 . \mathrm{Gk}, 43.60 . \mathrm{Cg}$

\section{INTRODUCTION}

Consider a horizontal linear array of sensors which is detecting coherent radiation from a source. If the medium is horizontally homogeneous, then the received signal is a cylindrical wave plus noise. The maximum-likelihood estimator of range assuming a cylindrical wave plus Gaussian noise has been studied by Hahn ${ }^{1}$ and Carter. ${ }^{2}$ The variance of the estimator due to additive noise is proportional to $R^{4} L^{-4}$, where $R$ is the range and $L$ is the array length. ${ }^{3}$ Inhomogeneities in the medium near the array distort the wave curvature, increasing the variance. Random variation of sound velocity. results in a loss of signal coherence which also increases variance. ${ }^{4}$ When $R$ is large, moreover, the curvature is almost impossible to measure.

There is another method for estimating the range to a distant source using a large aperture array. This approach makes use of the parallax effect. Suppose that the array is processed as a string of subarrays consisting of adjacent sensors. If each subarray gain is sufficiently large to resolve the source, then there will be a systematic measurable difference among the bearing estimates due to parallax. For the parallax method we need only assume that the received signal is a plane wave whose coherence distance is of the order of the subarray lengths. In other words, the signal must appear as a coherent plane wave to the subarrays, but not to the whole array. This assump-

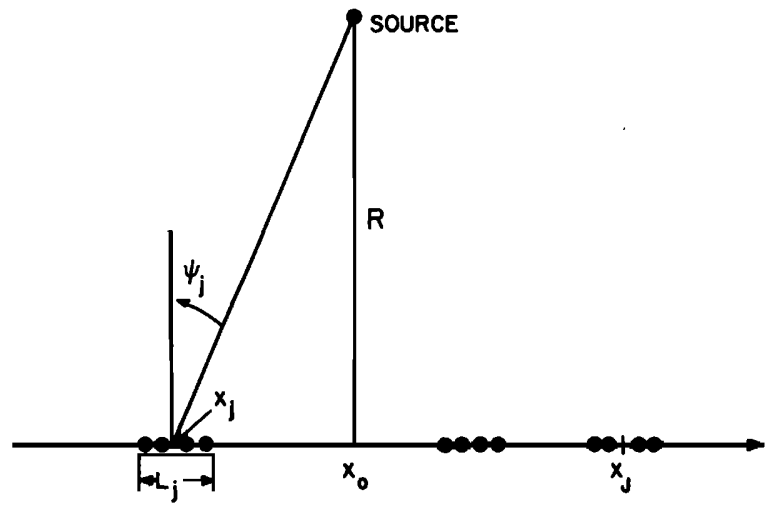

jth SUBARRAY

FIG. 1. Broadside source. tion about the signal is weaker than those made in the previously cited papers on focused arrays.

The range estimator using subarrays is presented in the next section. The method and its statistical properties also apply to a configuration of subarrays that cannot be connected together as a whole.

\section{PARALLAX RANGE ESTIMATION}

Suppose that the array consists of a line of $J$ linear subarrays. An array of $M=J M_{J}$ sensors can be electronically processed as $J$ subarrays of $M_{J}$ adjacent sensors. Assume that the signal at each subarray is a plane wave plus Gaussian noise with a signal-tonoise ratio denoted $\rho$, and we know the sign of the source bearing. To simplify a comparison with the statistical results obtained from the cylindrical model, assume that the medium is horizontally homogeneous and the wave is narrow band with center frequency $f$ and velocity $c$. Suppose that the $j$ th subarray is sampled for an interval $T=N \delta$. Let $x_{j}$ denote the coordinate of the center of the subarray on the array axis, and set the origin so that $\sum_{j=1}^{J} x_{j}=0$. Let $\hat{\psi}_{j}$ denote the maximumlikelihood estimator of bearing with respect to the axis perpendicular to the array at $x_{j}$ (Fig. 1). If $M_{j}$, the number of sensors in subarray $j$ is large, then the bearing that maximizes the energy of the output of a beamformer is maximum-likelihood. ${ }^{5}$ If $M_{j}$ or $N$ is large, ${ }^{6,7} \hat{\psi}_{j}=\psi_{j}+\left(\cos \psi_{j}\right)^{-1} \epsilon_{j}$ where the distribution of the error $\epsilon_{j}$ is approximately Gaussian with mean zero and variance $\sigma_{j}^{2}=\left(\gamma^{2} / M_{j} L_{j}^{2}\right) \operatorname{rad}^{2}$, where $L_{j}$ is the subarray length and

$$
\gamma^{2}=3 c^{2} / 2 N f^{2} \rho \pi^{2} \text {. }
$$

If the coherence distance of the noise is less than $x_{j+1}-x_{j}$, then $\epsilon_{j}$ and $\epsilon_{j+1}$ are uncorrelated.

Let $x_{0}$ denote the source's coordinate on the array

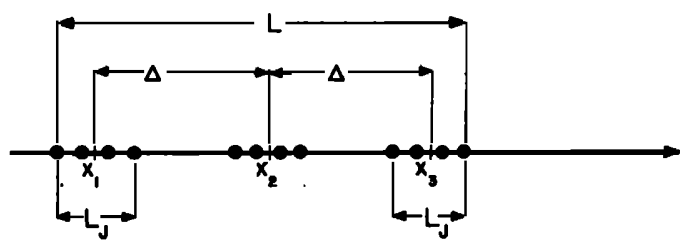

FIG. 2. Equally spaces subarrays. 
TABLE I. Statistical properties of $\hat{R}$.

\begin{tabular}{|c|c|c|c|c|c|}
\hline & $\begin{array}{c}\text { Mean bias } \\
\langle\hat{R} / R-1\rangle\end{array}$ & $\underset{\left\langle(\hat{R} / R-1)^{2}\right\rangle^{1 / 2}}{\text { rms error }}$ & $\begin{array}{c}\text { Mean } \\
\text { absolute error } \\
\langle|\hat{R} / R-1|\rangle\end{array}$ & $\begin{array}{c}\text { Mean } \\
\text { standard deviation } / R \\
\langle\sigma(\hat{R})\rangle / R\end{array}$ & $\begin{array}{c}\text { Asymptotic } \\
\text { standard deviation } / R\end{array}$ \\
\hline \multicolumn{6}{|c|}{$\sigma_{f}=0.5^{\circ}$} \\
\hline$R=280, J=14$ & 0.03 & 0.18 & 0.13 & 0.17 & 0.16 \\
\hline$R=560, J=28$ & 0.01 & 0.12 & 0.10 & 0.12 & 0.11 \\
\hline \multicolumn{6}{|c|}{$\sigma_{j}=1.0^{\circ}$} \\
\hline$J=14$ & 0.10 & 0.50 & 0.30 & 0.49 & 0.32 \\
\hline$J=28$ & 0.08 & 0.32 & 0.22 & 0.31 & 0.23 \\
\hline
\end{tabular}

axis and let $R$ denote its distance from $x_{0}$, i.e., $R$ is the range perpendicular to the array axis. Then $\tan \psi_{j}=\left(x_{0}-x_{j}\right) / R$. Using the linear approximation to $\tan \hat{\psi}_{j}$ for small $\sigma_{j}, \tan \hat{\psi}_{j}=\tan \psi_{j}+\left(\cos \psi_{j}\right)^{-3} \epsilon_{j}$. Suppose that $R \gg L$ and $x_{1} \leqslant x_{0} \leqslant x_{j}$. Then $\left(\cos \psi_{j}\right)^{-3}=\left\{1+\left[\left(x_{0}\right.\right.\right.$ $\left.\left.\left.-x_{j}\right) / R\right]^{2}\right\}^{3 / 2} \simeq 1$ for each $j$. Consequently,

$$
\tan \hat{\psi}_{j}=a-b x_{j}+\epsilon_{j},
$$

where $a=x_{0} R^{-1}$ and $b=R^{-1}$.

The weighted least-squares estimator of the slope $b$,

$$
\hat{b}=\frac{-\sum_{j=1}^{J} M_{j} L_{t}^{2} x_{j}\left(\tan \hat{\psi}_{i}-J^{-1} \sum_{j=1}^{J} \tan \hat{\psi}_{j}\right)}{\sum_{j=1}^{J} M_{j} L_{j}^{2} x_{j}^{2}}
$$

is maximum-likelihood since the $\epsilon_{j}$ 's are independent Gaussian errors. Moreover $\hat{b}$ is Gaussian with mean $R^{-1}$ and variance $\left.\gamma^{2} \sum_{j=1}^{J} M_{j} L_{j}^{2} x_{j}^{2}\right)^{-1}$. If $\gamma / L_{j}$ is small, this approximation of the mean and variance is good even if the $\epsilon_{j}$ are not Gaussian.

Consider the range estimator $\hat{R}=\hat{b}^{-1}$. To simplify the asymptotics, assume that the array consists of equally spaced subarrays of equal length $L_{J}$ with the same number of sensors, i.e., $x_{j+1}-x_{j}=\Delta, L_{j}=L_{j}$, and $M_{j}=M / J$ for each $j$ (Fig. 2). The array length is then $L=(J-1) \Delta+L_{J}$. Since $\sum_{j=1}^{J} x_{j}=0, x_{j}=[j-(J+1) / 2] \Delta$. Thus, $\sum_{f=1}^{J} M, L_{f}^{2} x_{j}^{2}=M L_{J}^{2} \Delta^{2}(J-1)(J+1) / 12$. Using Taylor's formula,

$$
\hat{R} / R=1-R(\hat{b}-b)+R^{2}(\hat{b}-b)^{2}+O\left[R^{3}(\hat{b}-b)^{3}\right] .
$$

Assume that $\gamma R \ll M^{1 / 2} L_{J} J \Delta$. Then the higher order terms in (4) are small and

$$
\begin{aligned}
(E \hat{R}) / R & =1+R^{2} E(\hat{b}-b)^{2} \\
& \simeq 1+\left[12 \gamma^{2} R^{2} / M L_{J}^{2} \Delta^{2}\left(J^{2}-1\right)\right] .
\end{aligned}
$$

From (4) the variance of $\hat{R} / R$ is

$$
\sigma^{2}(\hat{R}) / R^{2} \simeq 12 \gamma^{2} R^{2} / M L_{J}^{2} \Delta^{2}\left(J^{2}-1\right),
$$

which is greater than the square of the bias $E \hat{R} / R-1$ given the condition on $\gamma R$.

If $L$ is fixed, it is clear from (6) that the variance is minimized by using two abutting subarrays with $L_{J}=L / 2$ and $\Delta=L / 2$. In this case, $\sigma(\hat{R}) / R \simeq 8 \gamma M^{-1 / 2} L^{-2} R$. The optimal Hahn-Carter estimator ${ }^{8}$ has a proportional standard deviation of $\left[8 /(3)^{1 / 2}\right] \gamma M^{-1 / 2} L^{-2} R$. Thus my asymptotic standard deviation is $(3)^{1 / 2}$ times theirs, but they require the wave to be cylindrical for all values of $R$ whereas I assume a plane wave. A cylindrical wave becomes a plane wave as $R \rightarrow \infty$. Now let $\Delta=L_{J}$ $=L / J$ for $J \geqslant 3$. Then $\sigma(\hat{R}) / R \simeq 2(3)^{1 / 2} L^{-2} R$. My estimator is then less asymptotically efficient than the Hahn-Carter estimator for an equally spaced design.

\section{ARTIFICIAL DATA RESULTS}

The proportional bias, rms error, mean absolute error, and standard deviation of $\hat{R}$ were estimated for $R / J \Delta=20$ using $400 \hat{R}$ 's generated using Eqs. (2) and (3). An equally spaced subarray design was used with $\Delta=1, x_{0}=(J-1) / 2, J=14(R=280)$, and $J=28(R=560)$. The $\epsilon_{\text {, }}$ were computed using the same pseudorandom Gaussian generator that is programmed in the TI58 calculator, with $\sigma_{j}=0.5^{\circ}$, and $1^{\circ}$ for each $j$. The $95.5 \%$ confidence intervals for $\hat{\psi}_{j}$ are then $\psi_{j} \pm 1^{\circ}$ and $\psi_{j} \pm 2^{\circ}$, respectively. The results are given in Table $I$. The estimated $\sigma(\hat{R}) / R$ are close to their asymptotic values, with the greatest discrepancy for $\sigma_{j}=1^{\circ}$. These results support the proposition that the asymptotic results can be used as a design tool for processing a long array as a sequence of subarrays to estimate the range of a distant source when the array gain is large.

\section{ACKNOWLEDGMENT}

This work was supported by the Office of Naval Research under contract.

${ }^{1}$ W. R. Hahn, "Optimum Signal Processing for Passive Sonar Range and Bearing Estimation," J. Acoust. Soc. Am. 58, 201207 (1975).

${ }^{2}$ G. C. Carter, "Variance Bounds for Passively Locating an Acoustic Source with a Symmetric Line Array," J. Acoust. Soc. Am. 62, 922-926 (1977).

${ }^{3}$ W. J. Bangs and P. M. Schultheiss, "Space-Time Processing for Optimal Parameter Estimation," in Signal Processing, edited by J. W. R. Griffiths, P. L. Stocklin, and C. van Schooneveld (Academic, New York, 1973), pp. 577-590.

${ }^{4}$ M. M. Fitelson, " Broadside Bearing Response Pattern of a Line Array to a Cylindrical Wave in a Random Medium," J. Acoust. Soc. Am. 59, 525-527 (1976).

${ }^{5}$ M. J. Levin, “ Least-Squares Array Processing for Signals of Unknown Form," Radio Electron. Eng. 29, 213-222(1965).

${ }^{6} \mathrm{M}$. J. Hinich and P. Shaman, "Parameter Estimation for an R-Dimensional Plane Wave Observed with Additive Independent Gaussian Errors," Ann. Math. Stat. 43, 153-169 (1972).

7J. Capon and P. Green, "Statistical Accuracy of Data Used in Seismic Inversion," Geophys. J. R. Astron. Soc. 21, 373-386 (1970).

${ }^{8}$ From Eqs. (8), (10), and (11) in Ref. 2. 